\title{
Some integrals and series involving the Gegenbauer polynomials and the Legendre functions on the cut $(-1,1)$
}

\author{
Radosław Szmytkowski
}

\begin{abstract}
Atomic Physics Division, Department of Atomic Physics and Luminescence, Faculty of Applied Physics and Mathematics, Gdańsk University of Technology, Narutowicza 11/12, PL 80-233 Gdańsk, Poland

email: radek@mif.pg.gda.pl
\end{abstract}

June 7, 2018

\begin{abstract}
We use the recent findings of Cohl arXiv:1105.2735 and evaluate two integrals involving the Gegenbauer polynomials: $\int_{-1}^{x} \mathrm{~d} t\left(1-t^{2}\right)^{\bar{\lambda}-1 / 2}(x-t)^{-\kappa-1 / 2} C_{n}^{\lambda}(t)$ and $\int_{x}^{1} \mathrm{~d} t\left(1-t^{2}\right)^{\lambda-1 / 2}(t-x)^{-\kappa-1 / 2} C_{n}^{\lambda}(t)$, both with $\operatorname{Re} \lambda>-\frac{1}{2}, \operatorname{Re} \kappa<\frac{1}{2},-1<x<1$. The results are expressed in terms of the on-the-cut associated Legendre functions $P_{n+\lambda-1 / 2}^{\kappa-\lambda}( \pm x)$ and $Q_{n+\lambda-1 / 2}^{\kappa-\lambda}(x)$. In addition, we find closed-form representations of the series $\sum_{n=0}^{\infty}( \pm)^{n}[(n+\lambda) / \lambda] P_{n+\lambda-1 / 2}^{\kappa-\lambda}( \pm x) C_{n}^{\lambda}(t)$ and $\sum_{n=0}^{\infty}( \pm)^{n}[(n+\lambda) / \lambda] Q_{n+\lambda-1 / 2}^{\kappa-\lambda}( \pm x) C_{n}^{\lambda}(t)$, both with $\operatorname{Re} \lambda>-\frac{1}{2}$, Re $\kappa<\frac{1}{2},-1<t<1,-1<x<1$.
\end{abstract}

Key words: Special functions; Legendre functions; Gegenbauer polynomials; Fourier expansions

MSC2010: 33C55, 33C45, 33C05

\section{Introduction}

Recently, Cohl [1] has derived the integral formula

$$
\begin{array}{r}
\frac{n+\lambda}{\lambda} \int_{-1}^{1} \mathrm{~d} t \frac{\left(1-t^{2}\right)^{\lambda-1 / 2} C_{n}^{\lambda}(t)}{(z-t)^{\kappa+1 / 2}}=\mathrm{e}^{\mathrm{i} \pi(\lambda-\kappa)} \frac{\sqrt{\pi}(n+\lambda) \Gamma(n+2 \lambda)}{2^{\lambda-3 / 2} n ! \Gamma(\lambda+1) \Gamma\left(\kappa+\frac{1}{2}\right)}\left(z^{2}-1\right)^{(\lambda-\kappa) / 2} \mathfrak{Q}_{n+\lambda-1 / 2}^{\kappa-\lambda}(z) \\
\left(\operatorname{Re} \lambda>-\frac{1}{2}, \kappa \in \mathbb{C}, z \in \mathbb{C} \backslash(-\infty, 1]\right),
\end{array}
$$

where $C_{n}^{\lambda}(t)$ is the Gegenbauer polynomial, while $\mathfrak{Q}_{\nu}^{\mu}(z)$ is the associated Legendre function of the second kind. The integral (1.1) generalizes the Gormley's result [2]

$$
\begin{gathered}
\frac{n+\lambda}{\lambda} \int_{-1}^{1} \mathrm{~d} t \frac{\left(1-t^{2}\right)^{\lambda-1 / 2} C_{n}^{\lambda}(t)}{z-t}=\mathrm{e}^{\mathrm{i} \pi(\lambda-1 / 2)} \frac{\sqrt{\pi}(n+\lambda) \Gamma(n+2 \lambda)}{2^{\lambda-3 / 2} n ! \Gamma(\lambda+1)}\left(z^{2}-1\right)^{(\lambda-1 / 2) / 2} \mathfrak{Q}_{n+\lambda-1 / 2}^{1 / 2-\lambda}(z) \\
\left(\operatorname{Re} \lambda>-\frac{1}{2}, z \in \mathbb{C} \backslash(-\infty, 1]\right),
\end{gathered}
$$

which, in turn, is an extension of the celebrated Neumann's integral formula [3]

$$
\int_{-1}^{1} \mathrm{~d} t \frac{P_{n}(t)}{z-t}=2 \mathfrak{Q}_{n}(z) \quad(z \in \mathbb{C} \backslash[-1,1]),
$$

where $P_{n}(t)$ is the Legendre polynomial. The factor $(n+\lambda) / \lambda$ appearing in front of the integrals in Eqs. (1.1) and (1.2) (and also at some other places in the text) is only seemingly awkward and has been introduced to avoid the difficulty one otherwise encounters when $\lambda \rightarrow 0$. 
From Eq. (1.1) and from the closure relation for the Gegenbauer polynomials, which is

$$
\begin{array}{r}
\frac{2^{2 \lambda-1} \Gamma^{2}(\lambda)}{\pi} \sum_{n=0}^{\infty} \frac{n !(n+\lambda)}{\Gamma(n+2 \lambda)} C_{n}^{\lambda}(t) C_{n}^{\lambda}\left(t^{\prime}\right)=\frac{\delta\left(t-t^{\prime}\right)}{\left(1-t^{2}\right)^{(\lambda-1 / 2) / 2}\left(1-t^{\prime 2}\right)^{(\lambda-1 / 2) / 2}} \\
\left(\operatorname{Re} \lambda>-\frac{1}{2},-1<t, t^{\prime}<1\right)
\end{array}
$$

(here $\delta\left(t-t^{\prime}\right)$ is the Dirac delta function), one may deduce the summation formula [1]

$$
\begin{aligned}
\sum_{n=0}^{\infty} \frac{n+\lambda}{\lambda} \mathfrak{Q}_{n+\lambda-1 / 2}^{\kappa-\lambda}(z) C_{n}^{\lambda}(t) & =\mathrm{e}^{\mathrm{i} \pi(\kappa-\lambda)} \frac{\sqrt{\pi} \Gamma\left(\kappa+\frac{1}{2}\right)}{2^{\lambda+1 / 2} \Gamma(\lambda+1)} \frac{\left(z^{2}-1\right)^{(\kappa-\lambda) / 2}}{(z-t)^{\kappa+1 / 2}} \\
(\operatorname{Re} \lambda & \left.>-\frac{1}{2}, \kappa \in \mathbb{C},-1<t<1, z \in \mathbb{C} \backslash(-\infty, 1]\right) .
\end{aligned}
$$

The particular case of this relation with $\kappa=\lambda$ has been known before [4. p. 183]. For $\kappa=\lambda=1 / 2$, Eq. (1.5) reduces to the Heine's identity

$$
\sum_{n=0}^{\infty}(2 n+1) \mathfrak{Q}_{n}(z) P_{n}(t)=\frac{1}{z-t}, \quad(-1<t<1, z \in \mathbb{C} \backslash(-\infty, 1]) .
$$

In Sec. 2 of this communication, we show that one may use the relation in Eq. (1.1) to evaluate some further definite integrals involving the Gegenbauer polynomials. Furthermore, in Sec. 3, we exploit the identity (1.5) to determine closed-form representations of some series involving the Gegenbauer polynomials and the associated Legendre functions on the cut. While particular cases of the relations we arrive at in the present work, corresponding to specific choices of the parameters $\lambda$ and $\kappa$, may be found in the literature on special functions, we are not aware of any appearance of these relations in their most general forms derived below.

Throughout the paper, it is understood that

$$
\left(z^{2}-1\right)^{\alpha} \equiv(z-1)^{\alpha}(z+1)^{\alpha} \quad(|\arg (z \pm 1)|<\pi) .
$$

\section{Evaluation of some definite integrals involving the Gegen- bauer polynomials}

Let us assume that $\operatorname{Re} \kappa<\frac{1}{2}$. We proceed to the investigation of the limit of the formula in Eq. (1.1) as $z \rightarrow x \pm \mathrm{i} 0$, with $-1<x<1$. Exploiting the identities

$$
x \pm \mathrm{i} 0+1=1+x, \quad x \pm \mathrm{i} 0-1=\mathrm{e}^{ \pm \mathrm{i} \pi}(1-x) \quad(-1<x<1)
$$

and

$$
\mathrm{e}^{-\mathrm{i} \pi \mu} \mathfrak{Q}_{\nu}^{\mu}(x \pm \mathrm{i} 0)=\mathrm{e}^{ \pm \mathrm{i} \pi \mu / 2}\left[Q_{\nu}^{\mu}(x) \mp \frac{\mathrm{i} \pi}{2} P_{\nu}^{\mu}(x)\right] \quad(-1<x<1),
$$

where $P_{\nu}^{\mu}(x)$ and $Q_{\nu}^{\mu}(x)$ are the associated Legendre functions on the cut, we obtain

$$
\begin{aligned}
\frac{n+\lambda}{\lambda} \int_{-1}^{1} \mathrm{~d} t \frac{\left(1-t^{2}\right)^{\lambda-1 / 2} C_{n}^{\lambda}(t)}{(x \pm \mathrm{i} 0-t)^{\kappa+1 / 2}}= & \frac{\sqrt{\pi}(n+\lambda) \Gamma(n+2 \lambda)}{2^{\lambda-3 / 2} n ! \Gamma(\lambda+1) \Gamma\left(\kappa+\frac{1}{2}\right)} \\
& \times\left(1-x^{2}\right)^{(\lambda-\kappa) / 2}\left[Q_{n+\lambda-1 / 2}^{\kappa-\lambda}(x) \mp \frac{\mathrm{i} \pi}{2} P_{n+\lambda-1 / 2}^{\kappa-\lambda}(x)\right] \\
& \left(\operatorname{Re} \lambda>-\frac{1}{2}, \operatorname{Re} \kappa<\frac{1}{2},-1<x<1\right) .
\end{aligned}
$$

(The reason for imposing the above constraint on $\kappa$ is now clear: we have had to exclude a nonintegrable singularity at $t=x$ that otherwise occurs.) Since it holds that

$$
x \pm \mathrm{i} 0-t= \begin{cases}x-t & (t<x) \\ \mathrm{e}^{ \pm \mathrm{i} \pi}(t-x) & (t>x)\end{cases}
$$


we may split the integrals appearing on the left-hand side of Eq. (2.3) as follows:

$$
\begin{aligned}
\frac{n+\lambda}{\lambda} \int_{-1}^{1} \mathrm{~d} t \frac{\left(1-t^{2}\right)^{\lambda-1 / 2} C_{n}^{\lambda}(t)}{(x \pm \mathrm{i} 0-t)^{\kappa+1 / 2}}= & \frac{n+\lambda}{\lambda} \int_{-1}^{x} \mathrm{~d} t \frac{\left(1-t^{2}\right)^{\lambda-1 / 2} C_{n}^{\lambda}(t)}{(x-t)^{\kappa+1 / 2}} \\
& +\mathrm{e}^{\mp \mathrm{i} \pi(\kappa+1 / 2)} \frac{n+\lambda}{\lambda} \int_{x}^{1} \mathrm{~d} t \frac{\left(1-t^{2}\right)^{\lambda-1 / 2} C_{n}^{\lambda}(t)}{(t-x)^{\kappa+1 / 2}} \\
& \left(\operatorname{Re} \lambda>-\frac{1}{2}, \operatorname{Re} \kappa<\frac{1}{2},-1<x<1\right) .
\end{aligned}
$$

If we insert Eq. (2.5) into Eq. (2.3), and then equate separately terms corresponding to the choices of the upper and the lower signs on both sides of the resulting equation, we arrive at an inhomogeneous algebraic system for the two integrals standing on the right-hand side of Eq. (2.5). Solving this system, and using the well-known identity

$$
\Gamma(\zeta) \Gamma(1-\zeta)=\frac{\pi}{\sin (\pi \zeta)},
$$

we obtain

$$
\begin{array}{r}
\frac{n+\lambda}{\lambda} \int_{x}^{1} \mathrm{~d} t \frac{\left(1-t^{2}\right)^{\lambda-1 / 2} C_{n}^{\lambda}(t)}{(t-x)^{\kappa+1 / 2}}=\frac{\sqrt{\pi}(n+\lambda) \Gamma(n+2 \lambda) \Gamma\left(\frac{1}{2}-\kappa\right)}{2^{\lambda-1 / 2} n ! \Gamma(\lambda+1)}\left(1-x^{2}\right)^{(\lambda-\kappa) / 2} P_{n+\lambda-1 / 2}^{\kappa-\lambda}(x) \\
\left(\operatorname{Re} \lambda>-\frac{1}{2}, \operatorname{Re} \kappa<\frac{1}{2},-1<x<1\right)
\end{array}
$$

and

$$
\begin{aligned}
& \frac{n+\lambda}{\lambda} \int_{-1}^{x} \mathrm{~d} t \frac{\left(1-t^{2}\right)^{\lambda-1 / 2} C_{n}^{\lambda}(t)}{(x-t)^{\kappa+1 / 2}} \\
& =\frac{\sqrt{\pi}(n+\lambda) \Gamma(n+2 \lambda)}{2^{\lambda-3 / 2} n ! \Gamma(\lambda+1) \Gamma\left(\kappa+\frac{1}{2}\right)}\left(1-x^{2}\right)^{(\lambda-\kappa) / 2}\left\{Q_{n+\lambda-1 / 2}^{\kappa-\lambda}(x)-\frac{\pi}{2} P_{n+\lambda-1 / 2}^{\kappa-\lambda}(x) \cot \left[\pi\left(\kappa+\frac{1}{2}\right)\right]\right\} \\
& \quad\left(\operatorname{Re} \lambda>-\frac{1}{2}, \operatorname{Re} \kappa<\frac{1}{2},-1<x<1\right),
\end{aligned}
$$

respectively. The right-hand side of the latter equation may be simplified considerably after one exploits the known relation 1

$$
P_{\nu}^{\mu}(-x)=P_{\nu}^{\mu}(x) \cos [\pi(\nu+\mu)]-\frac{2}{\pi} Q_{\nu}^{\mu}(x) \sin [\pi(\nu+\mu)] \quad(-1<x<1)
$$

and the identity (2.6). This yields

$$
\begin{aligned}
& \frac{n+\lambda}{\lambda} \int_{-1}^{x} \mathrm{~d} t \frac{\left(1-t^{2}\right)^{\lambda-1 / 2} C_{n}^{\lambda}(t)}{(x-t)^{\kappa+1 / 2}} \\
& =(-)^{n} \frac{\sqrt{\pi}(n+\lambda) \Gamma(n+2 \lambda) \Gamma\left(\frac{1}{2}-\kappa\right)}{2^{\lambda-1 / 2} n ! \Gamma(\lambda+1)}\left(1-x^{2}\right)^{(\lambda-\kappa) / 2} P_{n+\lambda-1 / 2}^{\kappa-\lambda}(-x) \\
& \quad\left(\operatorname{Re} \lambda>-\frac{1}{2}, \operatorname{Re} \kappa<\frac{1}{2},-1<x<1\right) .
\end{aligned}
$$

Equation (2.10) may be also derived from Eq. (2.7) after in the latter one makes the simultaneous replacements $t \rightarrow-t$ and $x \rightarrow-x$, and subsequently exploits the property $C_{n}^{\lambda}(-t)=(-)^{n} C_{n}^{\lambda}(t)$.

Equations (2.7) and (2.10) constitute the result of this section. Their particular cases, corresponding to making the choices $\lambda=\frac{1}{2}$ and $\kappa=0$, may be found, albeit with the right-hand sides written in less compact forms, in Refs. [4, p. 261] and [6, p. 187].

\footnotetext{
${ }^{1}$ It is stated in Refs. [4 p. 170] and [5 p. 144] that the domain of validity of the relation displayed in our Eq. (2.9), and also of the counterpart expression for $Q_{\nu}^{\mu}(-x)$ in terms of $P_{\nu}^{\mu}(x)$ and $Q_{\nu}^{\mu}(x)$, is $0<x<1$. However, it is not difficult to show that if both relations hold on that interval, they must be valid for $-1<x \leqslant 0$ as well.
} 


\section{Evaluation of closed forms of some series involving the Gegenbauer polynomials and the associated Legendre functions on the cut}

Next, we shall draw inferences from the expansion (1.5), approaching the limit $z \rightarrow x \pm \mathrm{i} 0$, with $-1<x<1$. As in the preceding section, we assume that $\operatorname{Re} \kappa<\frac{1}{2}$ (recall that the formula in Eq. (1.5) is a consequence of Eq. (1.1) which in the limit discussed here loses its sense unless the above restriction is imposed on $\kappa$ ). With the use of Eqs. (2.1), (2.2) and (2.4), we arrive at

$$
\begin{aligned}
\sum_{n=0}^{\infty} \frac{n+\lambda}{\lambda}\left[Q_{n+\lambda-1 / 2}^{\kappa-\lambda}(x) \mp \frac{\mathrm{i} \pi}{2} P_{n+\lambda-1 / 2}^{\kappa-\lambda}(x)\right] C_{n}^{\lambda}(t) \\
=\frac{\sqrt{\pi} \Gamma\left(\kappa+\frac{1}{2}\right)}{2^{\lambda+1 / 2} \Gamma(\lambda+1)}\left(1-x^{2}\right)^{(\kappa-\lambda) / 2} \times\left\{\begin{array}{lr}
(x-t)^{-\kappa-1 / 2} & (-1<t<x<1) \\
\mathrm{e}^{\mp \mathrm{i} \pi(\kappa+1 / 2)}(t-x)^{-\kappa-1 / 2} & (-1<x<t<1)
\end{array}\right.
\end{aligned}
$$

Hence, subtracting or adding the two relations embodied in Eq. (3.1), we deduce that

$$
\begin{aligned}
& \sum_{n=0}^{\infty} \frac{n+\lambda}{\lambda} P_{n+\lambda-1 / 2}^{\kappa-\lambda}(x) C_{n}^{\lambda}(t) \\
&=\frac{\sqrt{\pi}}{2^{\lambda-1 / 2} \Gamma(\lambda+1) \Gamma\left(\frac{1}{2}-\kappa\right)}\left(1-x^{2}\right)^{(\kappa-\lambda) / 2} \times\left\{\begin{array}{cc}
0 & (-1<t<x<1) \\
(t-x)^{-\kappa-1 / 2} & (-1<x<t<1)
\end{array}\right. \\
&\left(\operatorname{Re} \lambda>-\frac{1}{2}, \operatorname{Re} \kappa<\frac{1}{2}\right)
\end{aligned}
$$

and

$$
\begin{aligned}
& \sum_{n=0}^{\infty} \frac{n+\lambda}{\lambda} Q_{n+\lambda-1 / 2}^{\kappa-\lambda}(x) C_{n}^{\lambda}(t) \\
&=\frac{\sqrt{\pi} \Gamma\left(\kappa+\frac{1}{2}\right)}{2^{\lambda+1 / 2} \Gamma(\lambda+1)}\left(1-x^{2}\right)^{(\kappa-\lambda) / 2} \times\left\{\begin{array}{cc}
(x-t)^{-\kappa-1 / 2} & (-1<t<x<1) \\
(t-x)^{-\kappa-1 / 2} \cos \left[\pi\left(\kappa+\frac{1}{2}\right)\right] & (-1<x<t<1)
\end{array}\right. \\
& \quad\left(\operatorname{Re} \lambda>-\frac{1}{2}, \operatorname{Re} \kappa<\frac{1}{2}\right) .
\end{aligned}
$$

If in Eqs. (3.2) and (3.3) we make the simultaneous replacements $t \rightarrow-t$ and $x \rightarrow-x$, we obtain two further summation formulas:

$$
\begin{aligned}
& \sum_{n=0}^{\infty}(-)^{n} \frac{n+\lambda}{\lambda} P_{n+\lambda-1 / 2}^{\kappa-\lambda}(-x) C_{n}^{\lambda}(t) \\
& =\frac{\sqrt{\pi}}{2^{\lambda-1 / 2} \Gamma(\lambda+1) \Gamma\left(\frac{1}{2}-\kappa\right)}\left(1-x^{2}\right)^{(\kappa-\lambda) / 2} \times \begin{cases}(x-t)^{-\kappa-1 / 2} & (-1<t<x<1) \\
0 & (-1<x<t<1)\end{cases} \\
& \left(\operatorname{Re} \lambda>-\frac{1}{2}, \operatorname{Re} \kappa<\frac{1}{2}\right), \\
& \sum_{n=0}^{\infty}(-)^{n} \frac{n+\lambda}{\lambda} Q_{n+\lambda-1 / 2}^{\kappa-\lambda}(-x) C_{n}^{\lambda}(t) \\
& =\frac{\sqrt{\pi} \Gamma\left(\kappa+\frac{1}{2}\right)}{2^{\lambda+1 / 2} \Gamma(\lambda+1)}\left(1-x^{2}\right)^{(\kappa-\lambda) / 2} \times \begin{cases}(x-t)^{-\kappa-1 / 2} \cos \left[\pi\left(\kappa+\frac{1}{2}\right)\right] & (-1<t<x<1) \\
(t-x)^{-\kappa-1 / 2} & (-1<x<t<1)\end{cases} \\
& \left(\operatorname{Re} \lambda>-\frac{1}{2}, \operatorname{Re} \kappa<\frac{1}{2}\right) .
\end{aligned}
$$

Some particular cases of the expansions (3.1)-(3.5), corresponding to specific choices of $\kappa$ and/or $\lambda$, may be found 2 in Refs. [4, pp. 182-183], [5, p. 166] and [7, pp. 341-342].

\footnotetext{
${ }^{2}$ In the second series in Sec. 4.5 .4 in Ref. [4 p. 182], $P_{m-1 / 2}^{\mu}(\cos \vartheta)$ should be replaced by $P_{m-1 / 2}^{\mu}(-\cos \vartheta)$. In the first formula in Ref. 4] p. 183], the constraint $x<\cos \varphi$ should be replaced by $-1<\cos \varphi<x<1$. Moreover, it follows from our Eq. (3.4) that the latter formula is valid at least for $\operatorname{Re} \nu>-\frac{1}{2}$.
} 


\section{Acknowledgments}

I thank Dr. Howard S. Cohl for drawing my attention to his work [1 and for a stimulating correspondence on the Legendre functions and related subjects.

\section{References}

[1] H. S. Cohl, On a generalization of the generating function for Gegenbauer polynomials, arXiv:1105.2735

[2] P. G. Gormley, A generalization of Neumann's formula for $Q_{n}(z)$, J. London Math. Soc. 9 (1934) 149

[3] F. Neumann, Entwicklung der in elliptischen Coordinaten ausgedrückten reciproken Entfernung zweier Puncte in Reihen, welche nach den Laplace'schen $Y^{(n)}$ fortschreiten; und Anwendung dieser Reihen zur Bestimmung des magnetischen Zustandes eines Rotations-Ellipsoïds, welcher durch vertheilende Kräfte erregt ist, J. Reine Angew. Math. (Crelle's J.) 37 (1848) 24 [reprinted in: Franz Neumanns gesammelte Werke. Dritter Band (Teubner, Leipzig, 1912) p. 439]. In the original printing of this paper, Neumann's given-name initial was typed incorrectly as $\mathrm{J}$ instead of $\mathrm{F}$.

[4] W. Magnus, F. Oberhettinger, R. P. Soni, Formulas and Theorems for the Special Functions of Mathematical Physics, 3rd ed. (Springer, Berlin, 1966)

[5] A. Erdélyi (ed.), Higher Transcendental Functions, Vol. 1 (McGraw-Hill, New York, 1953)

[6] A. Erdélyi (ed.), Higher Transcendental Functions, Vol. 2 (McGraw-Hill, New York, 1953)

[7] A. P. Prudnikov, Yu. A. Brychkov, O. I. Marichev, Integrals and Series, Vol. 3. Special Functions. Supplementary Chapters, 2nd ed. (Fizmatlit, Moscow, 2003) (in Russian) 